\title{
Psychological Models of Reading and Their Application to the Teaching of Reading in TCSOL
}

\author{
Cai Li
}

College of Chinese Language and Culture, Jinan University, Guangzhou, Guangdong, China (caili@ hwy.jnu.edu.cn)

\begin{abstract}
This article mainly introduces three kinds of the most representative models which respectively stand for all kinds of different summation of their systematic cognition about reading psychological processamong them since the 50's of the 20th century, and probes the value and the application tactics of these models on the teaching of reading in TCSOL.
\end{abstract}

Keywords - reading models, bottom up model, top down model, interactive model, the teaching of Chinese reading

\section{阅读心理模式及其在对外汉语阅读教学中的运用策略}

\author{
蔡丽 \\ 暨南大学华文学院, 广州, 广东, 中国
}

摘 要 本文探讨了二十世纪 50 年代以来心理学界提出的最有代表性的三种阅读模式在对外汉语阅读教学中的价值及运用策略。 本文认为, 在进行第二语言阅读教学时, 首先应进行自下而上阅读模式的训练。随着学生阅读水平的发展, 应专门进行自 上而下模式训练, 以培养预测、分析、推理能力和获取完整信息的能力。高级阶段, 将自下而上模式与自上而下模式结 合起来, 进行相互作用模式的训练。

关键词＼cjkstart阅读模式; 自下而上; 自上而下; 相互作用; 汉语阅读教学

\section{1. 引言}

阅读是人的一种高级复杂的认知行为, 是一种认知过 程、理解过程, 牵涉到许多复杂的心理问题。要提高阅读 教学的效率, 教师必须掌握有关的阅读理论, 只有在理论 指导下, 从 “教” 上下功夫, 才能使阅读教学符合语言认 知规律。

就研究对象来看, 阅读心理方面的研究理论一般都是 在研究第一语言阅读者阅读过程的基础上建立起来的, 虽 然第一语言阅读与第二语言阅读的心理过程有许多相似之 处, 但二者毕竟有所区别。张必隐和 Joseph H.Danks 在 1989 年进行的一项比较实验研究证明, 英语和汉语的阅读加工 策略不同, 并且认为 “两种语言在句法及语义层次上的加 工策略不同, 势必会对第二语言学习者在课文理解层次上 的策略的正常和正确使用产生影响” (张辉, 1999), 这已 得到阅读心理实验研究的进一步证实。对外汉语阅读教学, 在利用各种阅读理论指导教学实践的同时, 必须结合自身 特点, 建立符合自身规律的教学模式。

从二十世纪 50 年代以来, 心理学界提出过不少阅读模
式, 分别代表了心理学界对阅读心理过程系统认识的不同 概括, 本文介绍其中最有代表性的三种模式, 并探讨各种 模式在对外汉语阅读教学中的价值及运用策略。

\section{2. 关于阅读的三种模式}

\section{1 自下而上模式（bottom up model）}

“自下而上模式” 又称为 “文本驱动模式” 或 “数据 驱动模式”, 主要代表人物是高夫 (Gough), 也有人将该 模式称作 “高夫模式”。该模式认为阅读是一个精确的文字 解码的过程, 读者通过对字、词、词组等各级语言单位逐 层地进行连续、详尽的感知和辩认, 获得对文章的理解。 高夫认为阅读时, 读者首先通过视觉感知构成阅读材料的 印刷符号, 印刷符号通过视觉刺激传送到大脑, 大脑再对 这些符号进行辨认, 整个过程眼睛从左至右连续感知, 大 脑则对感知到的印刷符号进行从低级到高级地逐层加工。 这一模型把阅读过程分为肖像表征、字母的辨认、词的认 知、词在句子中的加工以及初级记忆等五个主要水平。任 
何一个水平上的信息处理都必须以低一级水平上的信息处 理为前提。也就是说, 要理解文章的意义, 必须先了解段 意; 要了解段意, 必须先了解句义; 而要了解句义, 对词 义的理解是关键。该模式认为读者对文章的理解完全取决 于对文章中词语的理解, 强调对低层次信息的处理过程。

在这种理论指导下的阅读教学往往将阅读理解的重点 置于阅读材料本身, 注重对字、词、语法的讲解, 强调逐 字逐句地进行阅读和阅读教学, 认为只有掌握了字词意义 及语法结构, 才能理解文章的意义, 因此, 在教学过程中 把扫除语言障碍作为首要问题。这种教学方法也就是通常 所称的“传统阅读教学法”, 也有人称其为 “讲解翻译模式”。

\section{2 自上而下模式（top down model）}

自上而下模式又叫 “概念趋进模式”, 是哥德曼 （Goodman）1967 年提出来的, 许多人又称之为 “哥德曼 模式”。Goodman (1967) 将阅读描述为 “心理语言学的猜 测游戏”, 他认为阅读并不是通过对所有语言成分进行精确 辨认而获取信息的过程, 而是一个选择的过程, 是一个意 义求证的过程。在阅读时读者根据自己头脑中已有的各种 知识对文章进行预测, 然后才去从印刷符号中获取样本来 验证自己的预测, 通过不断确证或修改, 在头脑中重建作 者所传达的信息, 最终获得对文章的理解。这一模式强调 阅读过程中读者思维活动的积极作用, 认为阅读并非读者 被动领会文章意义的过程, 文章的意义并不完全附于语言 外壳, 有效的阅读并不需要对读物中的所有字词作精确辨 认, 读者在阅读中只需从读物中获取能促使猜测和证实过 程顺利进行的 “最低数量” 的语言提示就行了。在阅读中, 读者必须利用已有知识积极主动去寻找意义线索, 对文章 不断进行预测、假设和验证，才能获得文章的深层意义。

近年来, 在外语阅读教学中采用的 “快速阅读训练” 就是自上而下模式的体现。这种阅读教学法将教学的着眼 点放在 “篇” 而不再是 “字句”, 强调语篇理解的重要性, 重视文章的主旨大义, 而不再将重心放在语言分析上。

\section{3 相互作用模式 (interactive model)}

相互作用模式是在综合自下而上模式和自上而下模式 的基础上吸取人工智能研究成果形成的, 其代表人物是鲁 梅哈特 (Rumelhart), 因此, 又叫 “鲁梅哈特模式”。这一 模式认为阅读时自下而上和自上而下两种加工在各个层次 同时发生, 阅读过程中的信息处理过程是在高层次的思维 活动与低层次的文字解码活动之间双向进行的。也就是说, 阅读是在文字符号线索与读者已有知识相互作用的基础上 进行的。读者要获得对文章意义的理解既需要获取视觉信 息和语言文字的外部特征, 也需要启动读者的各种知识,
包括语言知识、文化背景知识以及篇章结构知识等等。 Stanovich 认为二者之间还可以互相补偿, 当读者在某一层 次上有缺陷时, 会更多地依赖从更低层次或更高层次上获 取信息。

相互作用模式既强调运用已有的知识进行预测这种高 层次的加工, 又强调低层次的快速辨识文字符号的能力; 既重视阅读材料中文字信息的基础作用, 又强调读者的主 观能动性, 因而能比较全面地解释阅读过程中的各种现象。

相互作用模式的具体运行过程很复杂, 到目前为止, 阅读心理学家对阅读过程中具体的相互作用过程仍没有完 全统一的描述, 因而其总的理论框架虽可以对阅读教学起 一定指导作用, 但却无法解决一些具体问题。在第二语言 阅读教学中也没有形成与之相应的较有代表性的教学法。

\section{3. 阅读模式在对外汉语阅读教学中的运用策略}

在对外汉语阅读教学研究中, 对于在这些阅读心理模 式指导下形成的教学模式不乏评价。总体上看来, 对于在 自下而上模式基础上形成的讲解翻译模式, 大多持否定态 度, 认为 “长期以来人们对阅读的心理过程有一种误解, 认为阅读是精确地辨识字词的过程, 所以在对外汉语教学 中, 有时会片面强调逐字逐句的细读……其实, 这种做法 是不太科学的” (张惠芬, 2000)。对于在自上而下模式基 础上形成的快速阅读训练衰贬不一, 但持肯定意见的似乎 略多一些, “快速阅读可以大大增加学习者的阅读量, 扩大 阅读面, 丰富语感, 对于提高学生听、说、写等其它方面 的语言能力也有很大促进作用” (鲁宝元，1990); “快速阅 读不仅是一种迅速而全面地吸收有用信息的科学方法, 而 且也是一种高效的思维方法、记忆方法和有效的教学方法” (张惠芬, 2000)。对快速阅读训练持怀疑态度的学者认为 “考虑到外语学习的实际情况, 快速阅读只能说是一门不 实用的技术” (刘颂浩、林欢, 1996); “这种训练的弊病是, 在强调获得信息的同时, 读者失去了学习语言知识的机 会……快速阅读在阅读方法上所做的努力, 实质上是一种 治标不治本的做法……学生在课堂上学会的快读技术, 就 很难在自然阅读中应用” (刘颂浩, 2000)。相互作用模式 虽然没有形成有代表性的教学模式, 但在其基础上形成的 “图式理论”近年来却受到对外汉语教学工作者的重视 (储 诚志 1994, 张惠芬 2000, 朱建中 1997)。提倡用图式理论 指导阅读教学实际上是肯定了相互作用模式的积极作用。 “按照图式理论, 阅读一篇文章离不开 “自下而上”与 ‘自 上而下” 这一双重结构来进行解码” (朱建中, 1997)。

事实上，上述各种阅读模式及相应的教学模式都既有 合理的地方, 又有不符合规律之处。在自下而上模式理论 指导下进行的阅读教学有利于培养学生辨认文字符号的能 
力, 有利于巩固学生的语言知识, 使学生容易注意到新信 息, 让学生感觉学到了实在的东西。但这种方法过分强调 逐字逐句地阅读, 片面注重语言外部形式, 忽视了对阅读 材料整体意义的把握, 忽略了那些促进或阻碍学生阅读理 解的因素, 低估了学生在阅读过程中的积极能动作用。因 此, 在阅读中常常出现阅读材料中的字词都认识, 却不能 理解整个文章的意思, 且由于长期以来逐字逐句地讲解, 使学生养成逐字逐句阅读的习惯, 遇到生词就查字典, 因 此阅读速度很慢。

采用自上而下模式为理论依据的阅读教学把学生从拘 泥于逐字逐词地被动阅读中解放出来, 强调学生在阅读中 的积极作用, 强调学生已有背景知识对理解的重要作用, 对提高学生的阅读速度起到了极大的推动作用。但这种模 式过分强调高层次加工对低层次加工的指导作用, 过分强 调学生预测能力和背景知识的重要性, 而忽视了学生语言 能力的发展, 忽视了快速辨认词语、句子等低层次技能在 阅读理解中的基础作用。因此, 这类教学方法虽然有利于 提高学生阅读速度及理解能力, 但语言知识积累却受到阻 碍。从第二语言学习的角度来看, 第二语言阅读既是技巧 问题, 也是语言问题, 完全忽视语言问题的教学法显然有 失偏颇。

总之, 对于任何一种模式, 都不能彻底否认, 或全盘 肯定。在汉语作为第二语言的阅读教学中, 关键是做到根 据学生的实际情况和教学的客观需要, 全面权衡各种模式 的优缺点, 灵活地运用各种模式, 充分利用各种模式的优 势来进行教学。

\section{1 在进行第二语言阅读教学时, 教师首先应进行自下而} 上阅读模式的训练。

美国一些研究第一语言阅读教学的学者认为, 早期阅 读教学的任务是 “符号解码” (symbol decoding), 教学处 于 “学习识字” (learn to read) 阶段, 第二语言阅读教学也 是如此。学生对输入语言信息进行解码的能力, 在阅读理 解中起着最基本的作用, 语言的交际能力必须建立在扎实 的语言基础之上。英国贝尔法斯特女王大学托尼 - 里奇威

(Tony Ridgway) 博士 1997 年从事的一项关于外语阅读理 解中背景知识作用门限的研究表明: 如果读者的外语水平 处于未入门状况, 那么任何背景知识都无法对读者的阅读 理解发生作用。可见, 初级阶段的阅读教学更多地表现为 一种语言问题。因此, 自下而上的教学模式在初级阶段是 适用的。在这一阶段, 教师应努力实现以下两个目标:

第一, 训练学生对文字符号的辨识能力。自下而上模 式的代表人物之一 Laberge(1974)曾经从信息处理的角度研 究过阅读过程中注意力的作用, 他认为读者的注意力有限
的, 用于词语辨认处理的注意力越多, 给予词义的注意力 就越少, 因此, 阅读如果要达到熟练程度, 对语言低层次 技能的掌握 (如词汇辨认) 就必须达到自动的地步, 这样, 才能将注意力最大限度地集中于语义上。Gough 也认为熟 练读者的标志是准确、流利、迅速地辨认单词的能力。新 加坡的谢世涯、苏启祯 1992 年对新加坡高中二年级阅读理 解能力的调查数据表明, 学生阅读能力的 $52 \%$ 借助于单字 和词语的辨认能力。当然, 随着学生阅读水平的不断提高, 即使不作专门训练, 这种能力也会自然提高, 但其发展却 比较缓慢。佟乐泉、张一清在 1994 年研究一年级和二年级 外国留学生阅读能力发展时发现, “在学习 2 年汉语之后, 他们对两个字的词或词组的掌握已经达到了较熟练的程 度。……在每次呈现 4 个字的情况下，……还只是处在前 进过程中, 远没有达到熟练掌握的程度。” 因此, 在这一阶 段的阅读教学中, 教师除了要让学生掌握词义、句义外, 还应设计一些快速辨认词形的练习, 提高学生快速辨认词 语的能力。许多学者 (如鲁宝元 1990, 刘颂浩 1999, 张惠 芬 2000）根据自己的教学实践经验, 提出了一些快速辨认 词语的练习方式, 在教学中可用作参考。

第二, 熟悉汉语的语言表达方式, 逐步积累汉文化背 景知识。在阅读教学中, 人们往往根据阅读能力的发展将 教学分成不同阶段来谈, 不同阶段的教学重点各不相同。 尽管如此, 在教学中, 教师必须考虑到各个阶段之间并非 完全独立, 而是相互关联的。在前一阶段的教学中必须考 虑下一阶段的教学需要, 为下一阶段阅读能力的发展奠定 基础。运用自下而上模式阅读除了要培养学生辨认词语的 能力之外, 还要让学生熟悉汉语的语言表达方式, 并积累 相关的背景知识, 这些都是培养学生预测能力的基础。因 为 “不熟悉中国人心理和表达方法的人是很难依据上下文 预测、推理和概括的。” (李世之, 1997) 在教学中, 教师 应有意识地向学生介绍这些方面的知识, 使学生逐步建立 起与之相关的图式。

3.2 随着学生阅读水平的发展, 应专门进行自上而下模式训 练, 以培养学生的预测、分析、推理能力和获取完整信 息的能力。

阅读理解不是一个单纯的语言问题, 它同时是一个技 巧问题, 涉及语言之外的许多其他知识和能力。根据阅读 心理学家的研究, 阅读时既需要从阅读材料中获得的视觉 信息, 又需要由读者大脑中已有的知识经验提供的非视觉 信息。单一的自下而上模式只能获得对文章字面意义的理 解 (即 “字面理解” 层次, literal comprehension), 而无法 理解一些 “双关的、不确定的、模糊的信息”, 无法理解作 者隐含着的意义和观点(即 “推断性理解” 层次, inferential 
comprehension)。许多学生指出他们在阅读中常遇到的 “词 语都认识, 却不理解文章含义” 的现象, 而且对读过的内 容没有清晰的印象, 能记住的内容很少, 这些现象正是自 下而上这一模式的缺陷所造成的。所以, 中级阶段的阅读 教学应在进行自下而上模式训练的同时, 培养学生用自上 而下模式进行阅读的能力。自上而下模式以对高层次信息 的加工作为主要加工形式, 以阅读者主观能动作用为中心, 涉及选择、推论、预见、验证、修改等一系列过程。因此, 在进行自上而下模式阅读训练时, 应从以下几方面提高学 生的阅读能力:

第一, 积累汉语篇章结构知识, 培养快速阅读文章的 能力。运用自上而下模式来阅读文章时, 阅读不是从第一 句开始逐句逐句地读下去, 而是从高层次加工入手, 通过 选择最少的语言提示产生种种预测, 在最短的时间内在头 脑中重建作者所传达的信息。读者必须具备相关的篇章知 识, 才能了解一篇文章中哪些段落表达主题思想, 哪些段 落是具体陈述说明, 才能准确区分段落中的细节句与中心 句, 才能分清句子中哪些是已知信息, 哪些是新信息。如 果学生不了解篇章结构知识, 就根本不可能迅速从文章中 获取所需的信息提示, 而必然以逐字逐句的方式来阅读。 因此, 积累篇章结构知识是应用自上而下模式的必要条件 之一。当然, 篇章结构知识的分析应与培养学生快速阅读 的能力同时进行。快速阅读文章是许多学生在母语阅读中 就已经掌握了的一项技能。在汉语阅读教学中, 教师要做 的是让学生将这一技能运用到汉语阅读中来。虽然经过初 级阶段快速辨认词语的训练之后, 学生的辨词能力有可能 得到显著提高, 但不可能像母语阅读那样, 达到完全纯熟、 接近于机械反应式的程度, 因此, 学生很难在短期内自觉 地摆脱逐字逐句地阅读习惯。针对这种情况, 教师可采取 限时阅读、计时阅读等方式对学生进行快速阅读的训练, 也可以自己设计一些训练方法, 如张惠芬 (2000) 提出的 速读文章时目光注视的三种方法一一意群注视法、垂直注 视法、波浪注视法, 就很有参考价值。

第二, 训练学生利用文化背景知识进行预测的能力。 不同国家, 有着各自不同的价值观念、道德标准、思维方 式、风俗习惯以及行为准则等, 这些方面的特征在文章中 会体现出来, 有时甚至是支配文章的主线。掌握相关文化 背景知识, 在阅读中可以进行有效地预测。在阅读教学中, 教师应引导学生学会如何利用文化背景知识预测文章内 容。

3.3 高级阶段, 将自下而上模式与自上而下模式结合起来, 进行相互作用模式的训练。

经过前两个阶段的训练, 学生无论在语言能力, 还是
在阅读技巧的使用上, 都具备了一定的基础。此时, 教学 的重心应放在巩固、完善和补充现有知识和技能上, 包括 进一步扩大学生的词汇量和语法知识, 拓宽学生的知识面, 扩展学生大脑中的知识库, 增强学生假设、判断、分析、 推理、概括、归纳等逻辑思维能力以及细致的观察能力, 培养学生在阅读中跳跃障碍和难点, 灵活运用已有的各种 知识获取文章意义的熟练能力。要实现以上目标, 就必须 指导学生灵活地运用相互作用模式进行阅读。一些学者曾 指出, 相互作用模式具有高度的灵活性, 它对于 “自下而 上” 和 “自上而下” 两种信息加工方式在阅读过程中的相 互作用条件并没有限制。学生可以根据阅读的目的和方法, 以及自己对文章的难易程度与自己已有知识水平的判断, 确定是从高层次加工入手, 采取 “自上而下” 为主的策略, 还是从低层次信息获得开始, 逐步达到对文章细节的理解, 从而建立起新的图式。

\section{4. 结语}

在本文的论述中, 初、中、高级这三个阶段的划分是 以《汉语水平等级标准与语法大纲》的划分为标准的, 其 对象是三年制或四年制汉语专业的留学生。这类学生学习 汉语的时间充足, 对于每一种阅读模式都可以进行系统训 练, 第一学年主要训练学生用自下而上模式进行阅读, 第 二学年在继续进行自下而上模式训练的同时, 采用自上而 下模式为主要训练模式, 第三学年则以交互作用模式作为 主要训练模式, 这样可以为培养学生的阅读理解能力打下 坚实的基础。

\section{参考文献(References)}

[1] Li Shizhi, Reflections on the teaching of reading, Chinese Teaching in the World, vol.1, 1997.

[2] Lu Baoyuan, Fast reading training in TCSOL, Chinese Teaching In The World, vol.1, 1990.

[3] Liu Songhao, Lin Huan, Several problems in the teaching of reading, Language Teaching and Linguistic Studies, vol.1, 1996.

[4] Zhang Huifen, Visual consciousness, knowlege schemas and reading speed drills, Chinese Language Learning, vol.2,2000.

[5] Zhu Jianzhong, To explore the best teaching model of Chinese newspapers reading. Research on the Teaching of Language and Culture, 3rd.Sinolingua Co., Ltd., 1999.

[6] Zhang Hui. Strategy training in reading comprehension in TCSOL, Research on the Teaching of Language and Culture,3rd.Sinolingua Co., Ltd.,1999.

[7] Laberge, D.and S.J.Samuels, Toward a theory of automatic information processing in reading, Cognitive Psychology, vol.6,1974. 